\title{
SYSTEMS OF PARTICLES WITH SHIFTED MAGNETIC DIPOLES
}

\author{
M. Klinkigt ${ }^{1}$, R. Weeber $^{1}$, S. Kantorovich ${ }^{1,2}$ C. Holm ${ }^{1}$ \\ 1 Institute for Computational Physics, University of Stuttgart, \\ Pfaffenwaldring 27, 70569 Stuttgart, Germany \\ ${ }^{2}$ Ural State University, 51 Lenin av., 620000, Ekaterinburg, Russia
}

\begin{abstract}
In this contribution, we investigate the system of magnetic shifted dipoles using molecular dynamics simulations to elucidate the subject. We present an extensive study of the cluster formation in these systems both in three and two dimensions. It is shown that the more the dipole moment is shifted away from the particle centre the stronger it influences the microstructure of the system, both at low and room temperature. For larger shifts, the dimensionality of the system does not influence much the cluster structure.
\end{abstract}

Introduction. Magnetic colloids, namely, the systems of magnetic dipolar nanoparticles in nonmagnetic liquid carriers, are actively employed in various applications from engineering to medicine and have attracted the attention of scientists from many fields of natural science. Ferrofluids are employed as seals around rotating shafts [1] for delivering drugs to tumors [2], monitoring the dynamics of anti-body reactions [3], and for tomography [4]. Many of these applications are based on the ability to control the properties and dynamics of the fluid by an external magnetic field, or to probe them using sensors, making it important to understand the microstructure of ferrofluids itself and how it influences macroscopic properties.

The investigation of the microstructure of ferrofluids is a complex experimental and theoretical problem. It turned out that computer simulation techniques can nicely deal with microstructure analysis. Usually, in computer simulations, magnetic nanoparticles are modeled as hard or soft spheres, which additionally interact via the dipole-dipole potential:

$$
\begin{gathered}
U_{d}(i j)=-\frac{\mu_{0}}{4 \pi}\left[3 \frac{\left(\mathbf{m}_{i} \cdot \mathbf{r}_{i j}\right)\left(\mathbf{m}_{j} \cdot \mathbf{r}_{i j}\right)}{r^{5}}-\frac{\left(\mathbf{m}_{i} \cdot \mathbf{m}_{j}\right)}{r^{3}}\right], \\
\mathbf{r}_{i j}=\mathbf{r}_{i}-\mathbf{r}_{j}, \quad\left|\mathbf{r}_{i j}\right|=r,
\end{gathered}
$$

where $\mu_{0}$ is the vacuum permeability and $\mathbf{m}_{i}$ and $\mathbf{m}_{j}$ are the dipole moments of the particles, respectively. In other words, the so-called coarse-grained approach is employed. In this way, systems with different geometries, densities and interaction strengths have been investigated by many authors for more than 15 years. Here we provide only few of these works and address references therein [5-11].

As many of the magnetic fluid properties are well understood by now, various magnetic nanoparticles and colloids that deviate in one or another way from the spherical shape have been examined in recent years. Some examples are dumbbells, i.e. two overlapping spheres of opposite charges [12], magnetic core-shell particles [13], elongated ferroparticles [14], and colloidal particles with a magnetic cap [15]. In [16], we introduced and examined the ground states of a two-dimensional model system with magnetic particles, in which the dipole moment was shifted from the 
centre of mass towards the particle's surface. We called such particles "sd-particle" to abbreviate the term "shifted dipole particles". Although sd-particles are an abstract model, they show structures similar to those found for colloids with a magnetic cap [15] and they help us to further understand the peculiarities of the dipole-dipole interaction.

In this contribution, we continue the investigation of sd-particles and extend the dimensionality and the range of temperatures under study. Here, we use molecular dynamics simulations performed in ESPResSo [17]. In the following sections, first the model is presented (Sec. 1) and the computer simulations are described. In Sec. 2, we provide a detailed description of the microstructure of the three- and two-dimensional systems of shifted dipoles. We conclude our contribution with a brief summary and an outlook (Sec. 3).

1. Computer simulations. The sd-particle is a particle, in which the magnetic moment is shifted radially outwards from the centre of mass. The ratio between the distance from the centre of the particle to the position of the dipole $d$ and the particle radius $\sigma$ is the dimensionless shift of the dipole moment $\alpha$. In order to study the systems of shifted dipoles both at low and room temperatures, we use molecular dynamics simulations performed in ESPResSo. ESPResSo represents a unique package in the modern panorama of classical molecular dynamics tools. Through the scripting interface, the software gives the user a complete control on the utilization of its different modules: wide range of interaction potentials, different thermostating algorithms, treatment of long-range electrostatics and magnetostatics in 3D, 2D and 1D periodic geometries, rigid body dynamics, hydrodynamic interactions via Lattice-Boltzmann or dissipative particle dynamics, etc. Due to the fact that the dipole moment is shifted from the center of mass, standard simulation approaches, being those of direct sum or dipolar P3M [18], would not give true forces, which act on one particle. To solve this problem, there is a feature called "virtual sites" implemented in ESPResSo. The virtual sites are the particles, for which forces and torques are calculated, but the positions and orientations at the next time step are not derived using a standard integration scheme. They depend on the orientations and positions of other non-virtual site particles. This feature is really useful to create an algorithm, which is able to handle the shifted magnetic dipole within a soft-sphere particle. From the point of view of the simulation routine, the sd-particle is composed of two particles: a "real particle" (r-particle) in the sense that the position and orientation are calculated using a standard integration scheme, and a virtual site particle (vs-particle). The r-particle is placed at the center of mass of the nanoparticle and it interacts with other particles from the same species via a Weeks-Chandler-Andersen (WCA) potential. The vs-particle is placed at the position of the magnetic moment within the r-particle. Thus, the algorithm for simulating the evolution of the sd-particle system is the following: (1) to calculate the forces and the torques acting on the vs-particle arising from the dipole-dipole interaction or/and from the interaction with an external magnetic field, using the dipolar P3M algorithm; (2) to calculate the forces acting on the r-particle due to the WCA-potential; (3) to transform the forces and the torques obtained at the first step into those acting on the r-particle; (4) to integrate the equation of motion of the r-particle and to place the r-particle in its new position; (5) to calculate the new position and the new orientation of the vs-particle. With this algorithm at hand, one can perform computer experiments with various sd-particle systems and characterise their microstructure. 
System of particles with shifted magnetic dipoles

(a)

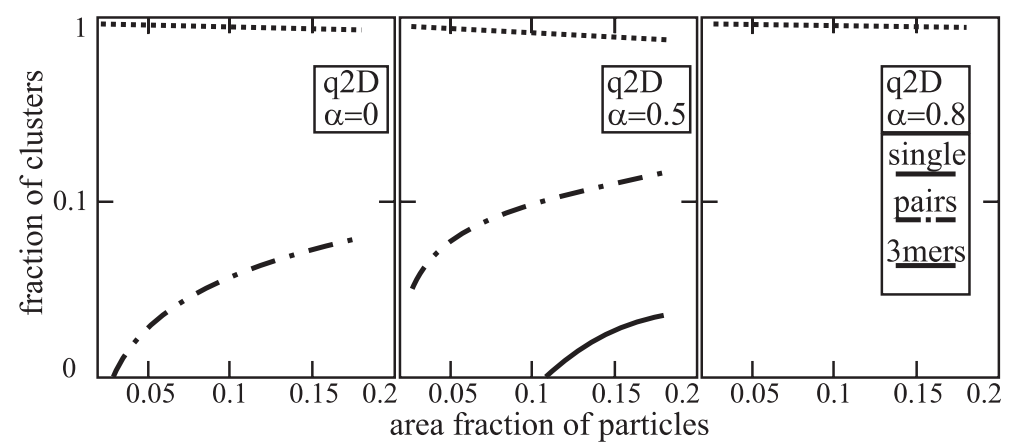

$\lambda^{*}=3$

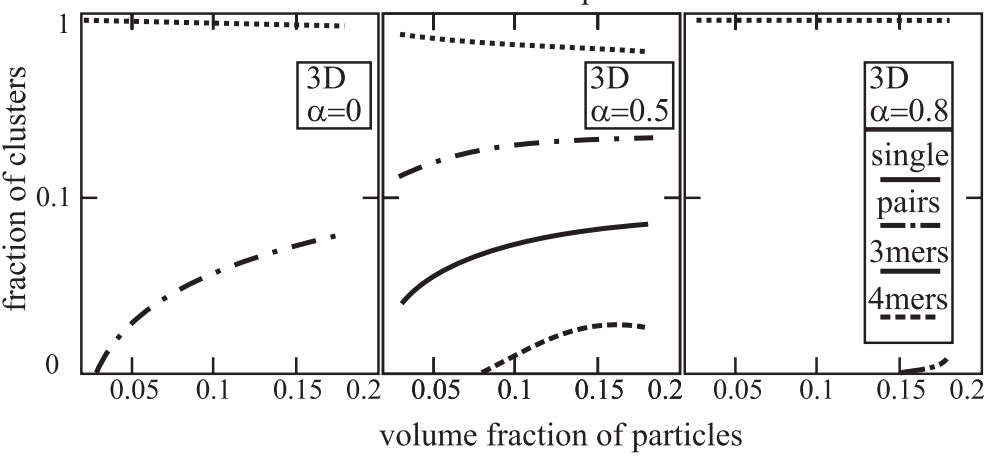

(b)

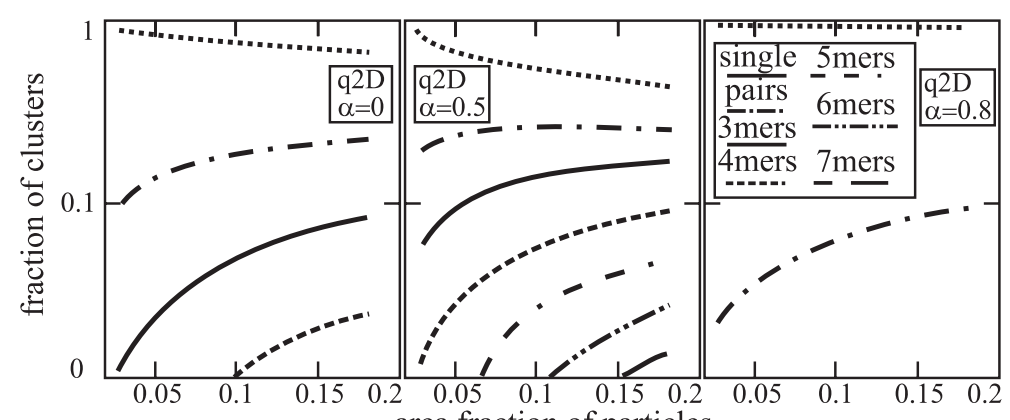

$\lambda^{*}=5$

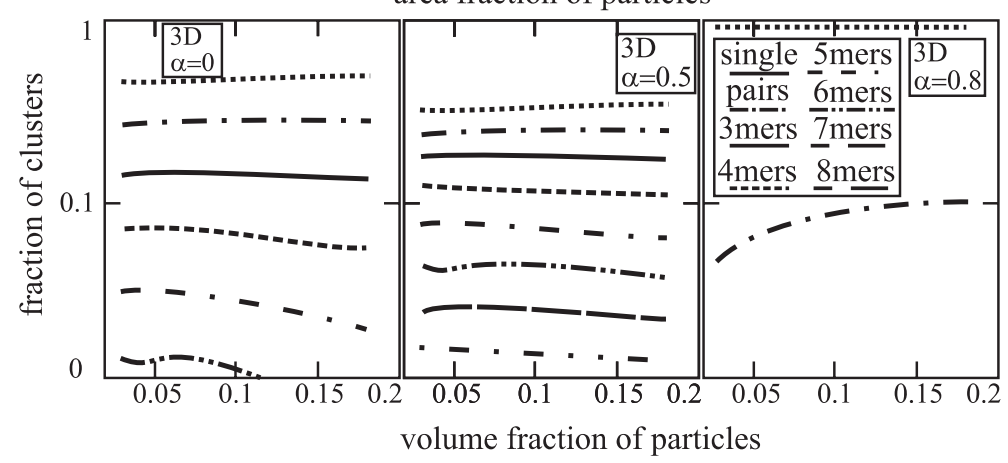

Fig. 1. The fraction of various clusters for $\lambda^{*}=3$ (left, $(a)$ ) and for $\lambda^{*}=5$ (right, (b)) versus the area and volume fraction of particles. A logarithmic scale is used for the ordinate axis. In the upper row, the results for quasi-two-dimensional systems are presented, in the lower row, the fractions in a bulk three-dimensional system are plotted. In the first column, the shift parameter $\alpha$ is zero; in the second column, $\alpha=0.5$; in the third column, the value of the shift is $\alpha=0.8$. All notations are provided in the legend. We use here the notation " $n$-mers" (with $n=3,4,5,6,7,8$ ) to abbreviate the fraction of the clusters of corresponding length. For example, the curve, which corresponds to "4mers" (short-dashed line) shows the fraction of the clusters composed of four sd-particles. 


\section{Klinkigt, R. Weeber, S. Kantorovich C. Holm}

2. Results and discussions. We have found out that the combination of the energy criterion and a criterion, which is able to detect triangular configurations, is a good approach to a cluster analysis algorithm. Upon introducing a cluster criterion, on can run the simulations and analyse the data. For that, $N=1000$ identical particles and their magnetic moments (radius $\sigma=1$ and magnetic moment $\mathbf{m}$, respectively) were randomly distributed in the simulation box. Periodic metallic boundary conditions were applied. The temperature $T$ was kept constant during the simulation using a standard Langevin thermostat with the relaxation time $\tau$. The time step was set to $2 \cdot 10^{-3} \tau$. After the equilibration time, which was of the order of $3 \cdot 10^{4}$ time steps, the positions and orientations of the particles were stored after each $10^{3}$ time steps.

Here, we analyse three- and quasi-two-dimensional systems. A quasi-twodimensional system is the one, in which the centres of mass of the particles are fixed in one plane, but the magnetic moments are free to rotate in all directions. We consider only the case of zero applied magnetic field. Various interaction parameters $\lambda^{*}$ (namely, $\lambda^{*}=3,5$ ), shift values $\alpha=0,0.5,0.8$ and different fractions $\rho=0.02-0.18$ per area/volume were examined. Here, we are interested in the fraction of clusters of a certain size in the system as a function of the area/volume fraction.

In Fig. 1 $(a, b)$, we plot the fraction of various clusters as a function of the particle area fraction (upper row, quasi-two-dimensional samples) and volume fraction (lower row, three-dimensional samples) for two values of $\lambda^{*}$, namely, in Fig. $1 a$ $\lambda^{*}=3$ and in Fig. $1 b \lambda^{*}=5$. From $(a)$ to $(b)$ one can see that the increase of $\lambda^{*}$ causes the enhancement of the aggregation for every shift, thus, the largest formed clusters for $(a)$ are composed of four particles (see, $\lambda^{*}=3,3 \mathrm{D}, \alpha=0.5$, solid line, 4-mers), however, for $\lambda^{*}=5$ even 8-mers, namely, clusters of eight sd-particles are observed (see, $\lambda^{*}=5,3 \mathrm{D}, \alpha=0.5$, dot-dot-dash-dot-dash-dot-dot line, 8-mers). From the upper row to the lower row one observes that the additional dimension also stimulates the clusterisation, especially at low densities. As for the shift value, then it is easy to observe that the less aggregated systems are those, for which $\alpha=0.8$ (last columns in $(a)$ and $(b)$ ). The largest clusters are observed for $\alpha=0.5$, and with no shift we obtain a standard system, where most ferroparticles remain single, and some pairs are formed with the increase of the volume/area fraction. It is worth mentioning that for the shift value $\alpha=0.5$ the behaviour of the systems seems to be quantitatively different from that at zero shift. So, what is the structure of the clusters for $\alpha>0$ at room temperatures?

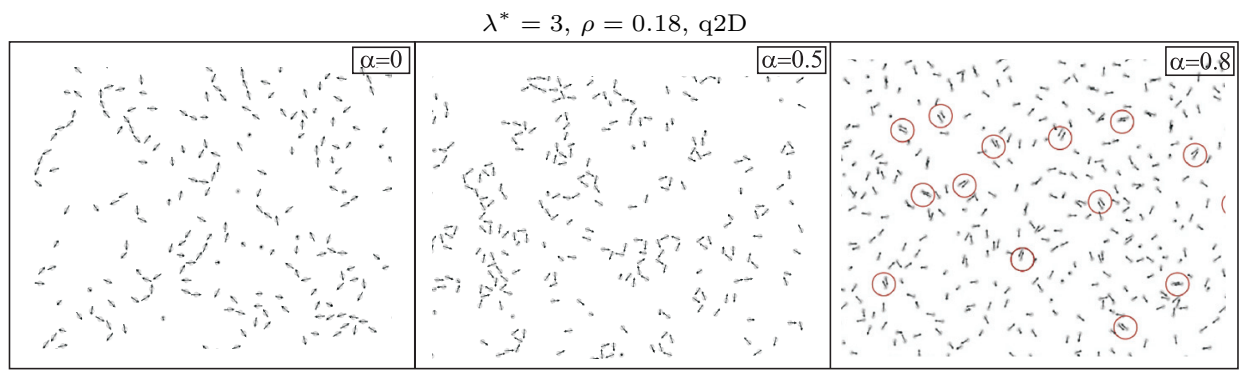

Fig. 2. Simulation snapshots of the quasi-two-dimensional system of sd-particles for $\lambda^{*}=5$ and area fraction $\rho=0.18$. From left to right the value of the shift $\alpha$ increases: $0,0.5$, and 0.8. One can see the obvious change of the microstructer from the chain aggregate for low shifts to antiparallel pairs for high shifts (indicated by circles). In the intermediate regime $(\alpha=0.5)$, the closed triangle-based large clusters can be found. 


\section{System of particles with shifted magnetic dipoles}

To answer this question, we provide Fig. 2, where simulation snapshot for $\lambda^{*}=5$ and volume fraction $\rho=0.18$ are presented. One can see that for $\alpha=0$ (the leftmost snapshot) the microstructure of the system is quite regular and chains are formed. For the shift value $\alpha=0.5$ (in the middle) the system seems far more aggregated, and already triangular and larger closed structures are observed. Let us point out that the ground state structure of three sd-particles for this shift value is a triangular configuration of particles, with the magnetic moments also aligned in a triangle. For the largest shift value $(\alpha=0.8$, the rightmost snapshot), the system looks weakly aggregated, and a new cluster type can be found. The system contains a significant amount of antiparallel pairs, whose structure agrees well with the ground state configuration.

3. Conclusions. We investigated the behaviour of the system of particles with magnetic dipoles shifted radially outwards. The extensive analysis of the microstructure at room temperature was done with the help of molecular dynamics simulations using the ESPResSo software package. Both bulk systems and monolayers were studied. We showed that in two dimensions independently of the shift value, the system is less aggregated than in the case of a bulk system. As for the shift influence, it turns out that at intermediate values of $\alpha$ the clusters are bigger than at high values of the shift. It happens so because of the cluster structure. Thus, at the intermediate shift the clusters predominantly have a triangular or a closed structure, whereas for the high values of shifts antiparallel pairs are mainly found. Currently we proceed with the investigation of the microstructure of sd-particle systems under the influence of an external magnetic field.

Acknowledgements. We are grateful for the financial support of MON s/c 02.740.11.0202, President RF Grant (MK-6415.2010.2) and Alexander von Humboldt foundation.

\section{REFERENCES}

[1] I. Anton, I. De Sabata And L. VÉKÁs. Application orientated researches on magnetic fluids. J. Magn. Magn. Mater., vol. 85 (1990), pp. 219-226.

[2] A. Lubbe, A.C. Alexiou, and C. Bergmann. Clinical applications of magnetic drug targeting. J. Surg. Res., vol. 95 (2001), pp. 200-206.

[3] B.Y. Ku, M.-L. Chan, Z. Ma And D.A. Horsley. Frequency-domain birefringence measurement of biological binding to magnetic nanoparticles. $J$. Magn. Magn. Mater., vol. 320 (2008), pp. 2279-2283.

[4] S.-J. Lee, J.-R. Jeong, S.-C.Shin, J.-C. Kim, Y.-H. Chang, Y.M. Chang AND J.-D.-D. KIM. Nanoparticles of magnetic ferric oxides encapsulated with poly(D,L latide-co-glycolide) and their applications to magnetic resonance imaging contrast agent. J. Magn. Magn. Mater., vol. 272-276 (2004), pp. 2432-2433.

[5] J.J. Weis And D. Levesque. Chain formation in low density dipolar hard spheres: A Monte Carlo study. Phys. Rev. Lett., vol. 71 (1993), pp. 2729 2732 .

[6] P.J. Camp and G.N. Patey. Structure and scattering in colloidal ferrofluids. Phys. Rev. E., vol. 62 (2000), pp. 5403-5408. 
[7] Z. Wang AND C. Holm. Structure and magnetization properties of polydispersed ferrofluids: A molecular dynamics study. Phys. Rev. E, vol. 68 (2003), pp. 041401-041411.

[8] C. Holm, A. Ivanov, S. Kantorovich, E. Pyanzina and E. Reznikov. Equilibrium properties of a bidisperse ferrofluid with chain aggregates: theory and computer simulations. J. Phys.: Condens. Matter, vol. 18 (2006), pp. 2737-2756.

[9] J.J. Cerdì, S. Kantorovich and C. Holm. Aggregate formation in ferrofluid monolayers: simulations and theory. J. Phys.: Cond. Matter, vol. 20 (2008), pp. 204125-204129.

[10] J. JoRdANOviC AND S.H.L. KLAPP. Field-induced layer formation in dipolar nanofilms. Phys. Rev. Lett., vol. 101 (2008), pp. 038302-038305.

[11] T. Prokopieva, V. Danilov, S. Kantorovich and C. Holm. Ground state structures in ferrofluid monolayers. Phys. Rev. E., vol. 80 (2009), pp. 031404-031417.

[12] G. Ganzenmueller and P.J. Camp. Vapor-liquid coexistence in fluids of charged hard dumbbells. J. Chem. Phys., vol. 126 (2007), pp. 191104-191107.

[13] A.P. Philipse, M.P.B. van Bruggen and P. Pathmamanoharan. Magnetic silica dispersions: preparation and stability of surface-modified silica particles with a magnetic core. Langmuir, vol. 10 (1994), pp. 92-99.

[14] F. Vereda, J. Vicente and R. Hidalgo-Alvarez. Physical properties of elongated magnetic particles: Magnetization and friction coefficient anisotropies. Chem. Phys. Chem., vol. 10 (2009), pp. 1165-1179.

[15] L. Baraban, D. Makarov, M. Albrecht, N. Rivier, P. Leiderer and A. ERBE. Frustration-induced magic number clusters of colloidal magnetic particles. Phys. Rev. E, vol. 77 (2008), pp. 031407-031412.

[16] S. Kantorovich, R. Weeber, J. J. Cerdà and C. Holm. Ferrofluids with shifted dipoles: Ground state structures. Soft Matter, DOI: 10.1039/c1sm05186e.

[17] H.-J. Limbach, A. Arnold, B. Mann and C. Holm. ESPResSo - An extensible simulation package for research on soft matter systems. Comput. Phys. Commun., vol. 174 (2006), pp. 704-727.

[18] J.J. Cerda, V. Ballenegger, O. Lenz, and C. Holm. P3M algorithm for dipolar interactions. J. Chem. Phys., vol. 129 (2008), pp. 234104-234123. 